\title{
AN EFFiCient CALl AdMission Control SCHEME FOR HANDLING HANDOFFS IN WIRELESS MOBILE NETWORKS
}

\author{
Alagu $S^{1}$, Meyyappan $T^{2}$ \\ ${ }^{1}$ Research Scholar, Department of Computer Science and Engineering \\ Alagappa University, Karaikudi, Tamilnadu, India \\ sivaalagu@hotmail.com \\ ${ }^{2}$ Professor, Department of Computer Science and Engineering \\ Alagappa University, Karaikudi, TamilNadu, India \\ meyslotus@yahoo.com
}

\begin{abstract}
Personal Communication Network $(P C N)$ is an emerging wireless network that promises many new services for the telecommunication industry. The proliferation of demands for extending wireless services to integrated services, which supports the transmission of data and multimedia information, has resulted in the need for broadband wireless systems that are able to provide service capabilities similar to those of wire line networks. The ATM cell - relay paradigm is one possible approach to provide broadband wireless transmission with PCN's using the ATM switching networks for interconnection of PCN cells. As traffic in these mobile cellular networks increases, Handoffs will become an increasingly important issue. As cell sizes shrink to accommodate an increasingly large demand of services, newer more efficient handoff schemes need to be used. In this paper, the authors describe the use of novel and efficient data structure which dynamically allocates guard channel for handoffs and introduces the concept of channel borrowing strategy. The proposed scheme allocates the guard channels for handoff requests dynamically, based on the traffic load for certain time period. A new originating call in the cell coverage area also uses these guard channels if they are unused. Our basic idea is to allow Guard channels to be shared between new calls and handoff calls. This approach maximizes the channel utilization. The simulation results prove that the channel borrowing scheme improves the overall throughput.
\end{abstract}

\section{KEYWORDS}

Guard Channel, Dynamic, Channel Borrowing, Handoff, Handover, ATM, QoS

\section{INTRODUCTION}

The field of wireless/mobile communication is at an interesting juncture in its development. The phenomenal worldwide growth of cellular telephony clearly demonstrates the fact that users place significant value on portability as a service feature. As end-user applications migrate towards Internet/WWW and broadband multimedia, it would be reasonable to expect strong consumer demand for wireless extensions to such services. However, this inevitable migration of mobile services to include integrated services such as data, voice, images and video represents a nontrivial architectural and technical challenge for the historically telephony-centric wireless industry. In particular, effective support to mobile users engaging in multimedia information services will require the communication path to provide sufficient buffers that cater to the change of bandwidth when the users moves from one location to another.

DOI : 10.5121/ijans.2012.2303 


\subsection{Cellular Networks}

A cellular network allows cellular subscribers to wander anywhere in the region and remain connected to the Public Switched Telephone Network (PSTN) via their mobile terminals. A cellular network has a hierarchical structure and it is formed by connecting major components mentioned below:

$>$ Mobile Terminals (MT)

$>$ Base Station (BS)

$>$ Mobile Switching Centre (MSC)

The Mobile Terminal is a hand held device, which the end-user uses to keep connected to a base station (home or away).

The Base Station serves a cell, which could be a few kilometers in diameter as shown in Figure 1. The cell is a part of a larger region, which has been partitioned into smaller regions such that there is a base station serving each cell. The cells when grouped together form a cluster which is depicted in Figure 2.

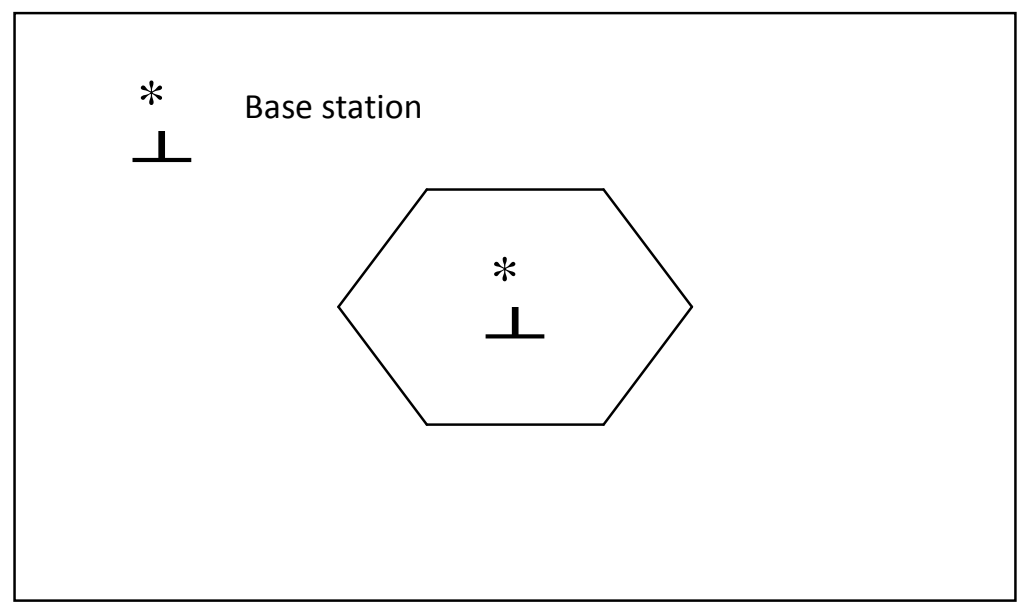

Figure 1. Base Station (BS)

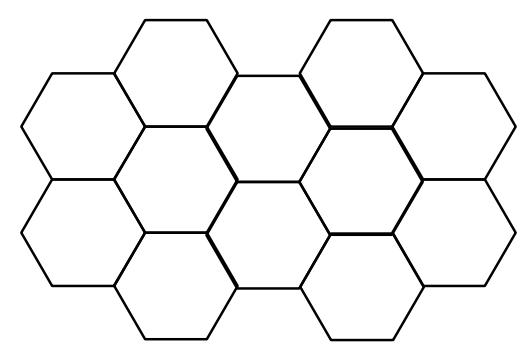

Figure 2. Cluster of Cells

All BSs within a cluster are connected to a Mobile Switching Centre (MSC) using landlines (coaxical cable or optical fibre). Each MSC of a cluster is then connected to the MSC of other 
clusters and a PSTN main switching center. The MSC stores information about the subscribers located within the cluster and is responsible for directing calls to them. Figure 3 shows the structure of the network explained above.

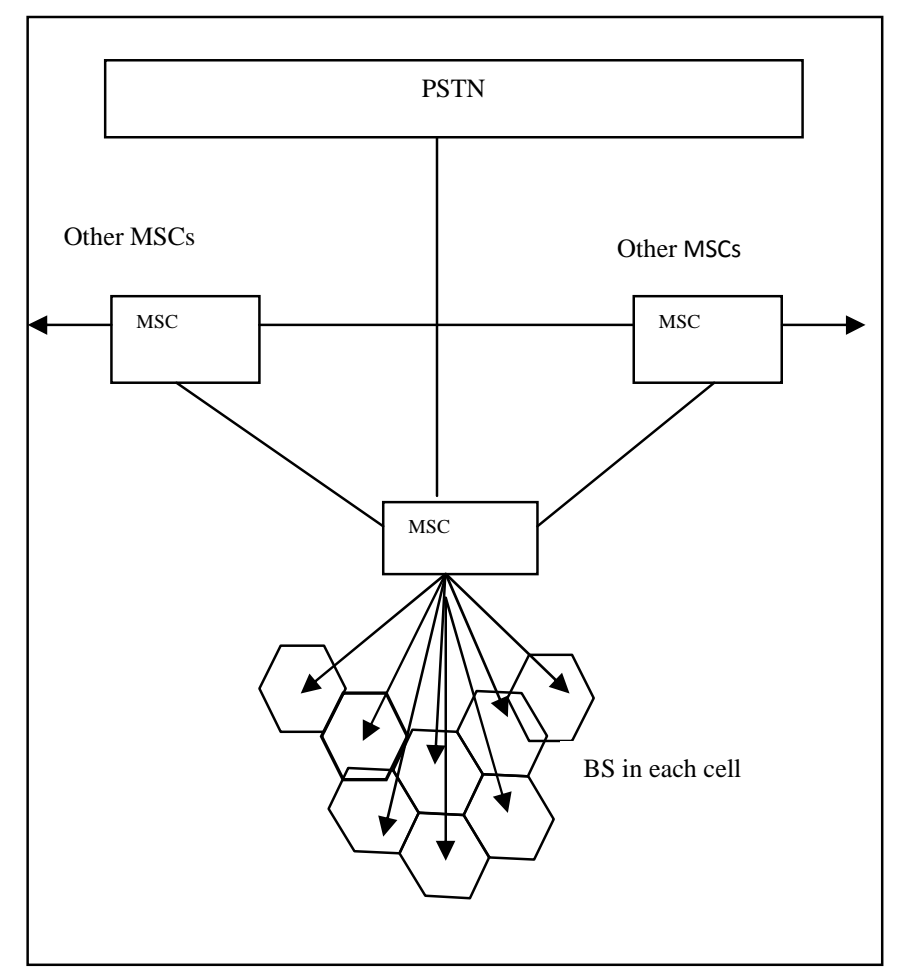

Figure 3. PSTN Connection with the MSCs

Hexagonal shaped cells shown in Figure 1 are artificial and cannot be generated in the real world. However this shape is chosen to simplify planning and design of a cellular system as hexagons fit together without any overlap or gap between them. Another advantage of using hexagons is that it approaches a circular shape, which is the ideal power coverage area.

The size of the cell largely depends on the area in which the cell is located. Generally, rural areas have fewer subscribers compared to urban areas. So in urban area more channels are needed to accommodate the larger number of subscribers.

\subsection{Allowing Mobility of Subscriber}

In order to allow mobility to a subscriber

The cellular network has to have the ability to track the subscriber down when a call is made to them.

$>$ Allow the subscriber to make calls while they are not in their home.

Tracking down is only possible if 
- The system maintains information about the location of the subscriber's mobile terminal.

- The mobile terminal knows the appropriate channels to await signals from the system.

In order for both the cellular system and the mobile terminal to have this required knowledge, there are two main procedures to follow when the subscriber turns on a mobile terminal in any location.

They are:

\section{- Searching for channels}

There are two channels that are searched by the mobile terminal which is explained in table 1 .

Table 1. Channels used by Mobile Terminal

\begin{tabular}{|l|l|l|}
\hline \multicolumn{2}{|c|}{ Channel } & \multicolumn{1}{|c|}{ Purpose } \\
\hline $\begin{array}{l}\text { Strong Dedicated Control } \\
\text { Channel (DCC) }\end{array}$ & $\begin{array}{l}\text { A channel used for the transmission of digital } \\
\text { control information from a base station to the } \\
\text { mobile terminal or vice versa. }\end{array}$ \\
\hline Strong Paging Channel & $\begin{array}{l}\text { A channel used by the MSC for seeking the } \\
\text { mobile terminal when a call made to it. }\end{array}$ \\
\hline
\end{tabular}

- Register to the NEAREST Base Station

The mobile terminal registers by sending identification number as mentioned in table 2 .

Table 2. Identification number used for registration

\begin{tabular}{|c|c|c|c|}
\hline \multicolumn{3}{|c|}{ Id } & Purpose \\
\hline $\begin{array}{l}\text { Mobile I } \\
\text { (MIN) }\end{array}$ & tificatic & Number & The unique network id of the mobile terminal. \\
\hline $\begin{array}{l}\text { Electronic } \\
(\mathrm{ESN})\end{array}$ & Serial & Number & $\begin{array}{l}\text { This is assigned to the mobile terminal by the } \\
\text { manufacturer. }\end{array}$ \\
\hline
\end{tabular}

The MIN and ESN are used by the MSC for access validation. This involves checking with the information stored in the home base station of the subscriber. Information about the mobile terminal and its current position is stored in the Mobile Switching Centre (MSC). Both the information stored in the MSC and the paging channel is used by the MSC to direct any arriving call to the appropriate subscriber. When the subscriber makes calls, they are allowed to be anywhere within the network as this involves the mobile terminal accessing base station - which could be located in any of the cells throughout the network. This base station can use the information it has retrieved from the home base station of the subscriber to direct their calls. 


\subsection{Moving the mobile terminal from one cell to another}

During a call, the base station would monitor the signal level from the mobile terminal. When the mobile terminal is moved into a new cell, the signal level will fall to a critical value causing the base station to inform the MSC about this event. The MSC would instruct all the surrounding base stations to measure the mobile terminal's signal level and transfer the control to the base station receiving the strongest signal level. This is known as hand-over or hand off and occurs within $400 \mathrm{~ms}$, so the subscribers are hardly aware of the break. Now registration is done again with the new BS. Location information stored in the MSC about this mobile terminal is updated. If the mobile terminal is moved into a cell belonging to a different cluster it would also have to register with the new MSC.

\subsection{Asynchronous Transfer Mode (ATM)}

The bandwidth requirements for data traffic within commercial organizations have been increasing steadily for some time, both in the local area networks and in wide area networks. Workstations have been used to introduce multimedia applications to the desktop, including components of voice, video and image, besides growing amount of data. This development requires networks of greater bandwidth than commonly present today with the capability of handling multiservice traffic on the same network.

The Asynchronous Transfer Mode (ATM) is being developed as a high speed networking technique for public networks capable of supporting many classes of traffic. Asynchronous Transfer Mode (ATM) has been accepted universally as the transfer mode of choice for Broadband Integrated Services Digital Networks (B-ISDN).

ATM is a high-speed, packet switching technique that uses short fixed length packets called cells. Fixed length cells simplify the design of an ATM switch at the high switching speeds involved. The selection of a short fixed length cell reduces the delay and most significantly the jitter (variance of delay) for delay-sensitive services such as voice and video. ATM is capable of supporting a wide range of traffic types such as voice, video, image and various data traffic. ATM transmits switches and multiplexes information in fixed-length cells. The length of a cell is 53 bytes, consisting of a 5 -byte cell header and 48 bytes of data.

\begin{tabular}{|l|c|}
\hline Header & PAYLOAD \\
\hline 5 Bytes & 48 Bytes \\
\hline
\end{tabular}

Table 3. ATM Cell

\subsubsection{Quality of Service (QoS)}

ATM networks are thought to transmit data with varying characteristics. Different applications need various Qualities of Service (QoS). Some applications like telephony may be very sensitive to delay, but rather insensitive to loss, whereas others like compressed video are quite sensitive to loss. The ATM Forum specified several Quality of Service (QoS) categories:

- CBR (Constant Bit Rate)

- VBR (Variable Bit Rate)

- ABR (Available Bit Rate)

- UBR (Unspecified Bit Rate) 


\section{Constant Bit Rate (CBR)}

During a connection setup CBR reserves a constant amount of bandwidth. This service is conceived to support applications such as voice, video and circuit emulation, which requires small delay variations (jitter). The source is allowed to send the negotiated rate any time and for any duration. It may temporarily send at a lower rate as well.

\section{Variable Bit Rate (VBR)}

VBR negotiates the Peak Cell Rate (PCR), the Sustainable Cell Rate (SCR) and the Maximum Burst Size (MBS). VBR sources are bursty. Typical VBR sources are compressed voice and video. These applications require small delay variations (jitter).

\section{Available Bit Rate (ABR) and Unspecified Bit Rate (UBR)}

$A B R$ and UBR services should efficiently use the remaining bandwidth, which is dynamically changing in time because of VBR service. Both are supposed to transfer data without tight constraints on end-to-end delay and delay variation. Typical applications are computer communications, such as file transfers and e-mail.

UBR service provides no feedback mechanism. If the network is congested, UBR cells may be lost.

An ABR source gets feedback from the network. The network provides information about the available bandwidth and the state of congestion. The source's transmission rate is adjusted in function of this feedback information. This more efficient use of bandwidth alleviates congestion and cell loss. For ABR service, a guaranteed minimum bandwidth (MCR) is negotiated during the connection setup negotiations.

\subsection{Wireless ATM}

In recent years there has been an increasing trend towards personal computers and workstations becoming portable and mobile. These ever-increasing groups of mobile users have been demanding access to network services similar to their tethered counterparts. The desire to provide universal connectivity for these portable and mobile computers and communication devices is fueling a growing interest in wireless packet networks. At the same time, wire line communication networks have been undergoing a revolutionary change themselves with the introduction of Asynchronous Transfer Mode (ATM) based Broadband Integrated Services Digital Network (B-ISDN) which can provide Quality of Service (QoS) guarantee. Given these rapid advancements, the communication networks of today are employing wireless media in the local area and utilizing wire line physical media in the metropolitan and wide area environment.

To support multimedia applications in wireless systems, it is necessary to construct a wireless networking infrastructure that can support QoS guarantees essential to provide broadband services. Since ATM is the standard for wire line broadband networks, it has generally been agreed that broadband services are best provided to wireless users by exploiting ATM in wireless systems. However, since the characteristics of the wireless communication channels (e.g., high bit error rate and user mobility) are significantly different from those of wire line channels, solutions that are designed for wire line networks cannot be expected to work for wireless environments. 


\subsection{Personal Communication Network (PCN)}

Personal Communication Network (PCN) is an emerging wireless network that promises many new services. With the availability of the interface cards, mobile users are no longer required to be confined within a static network premise to get network access. Mobile users may move from one place to another and yet maintain transparent network access through wireless links. Information exchanged between users, may be bi-directional, which includes but not limited to voice, data and image, irrespective of location and time while permitting users to be mobile.

In a PCN, the covered geographical area is typically partitioned into a set of microcells. Each microcell has a base station to exchange radio signals with wireless mobile terminals. Due to the limited range of wireless transceivers, mobile users can communicate only with the base stations that reside within the same microcell at any instance. When a mobile terminal, engaged in a cell or data transfer, moves out of the coverage area of the base station it is communicating with, the call must be transferred to another base station of the new cell; otherwise connection is lost. This transfer is known as handoff, is transparent to the mobile users. The number of handoffs during a call will increase as the cell radii decrease, thus affecting the quality of service. As a result of the increase in processing load due to demand for service and fast handoffs to mitigate the propagation effect, a high speed backbone network for the PCN to connect base stations is required. The ATM technology, which has recently emerged to be a predominant switching technology, is suited to be an infrastructure to interconnect the base stations of the PCN. Thus the given geographical area is partitioned into a set of disjoint clusters, each of which consists of a set of microcells. Each microcell has a base station to serve the mobile terminals within the cell. An ATM switch is allocated within each cluster and each of the base stations in this cluster is connected to one of the ports of this ATM switch. The ATM switch offers the services of establishing / releasing channels for the mobile terminals in the cluster. Two neighboring clusters can be interconnected via the associated ATM switches. The links between the ATM switches are called Backbone Links, and the links between an ATM switch and base stations are called Local Links. Each base station has a given number of radio channels for calls generated within its cell. Mobile hosts engaging in a call or data transfer within the same cluster will consume two local channels, one for each local link, between the base stations and the associated switch and one radio channel. For intercluster communication, backbone links will be allocated in addition to the local links and radio channel, and the channels occupied will depend on the communicating path being assigned.

\section{HANDOFF}

Handoff is the mechanism that transfers an ongoing call from one cell to another as a user moves through the coverage area of a cellular system. The handover process is initiated by the issuing of handover request. The power received by the MS from BS of neighboring cell exceeds the power received from the BS of the current cell by a certain amount. This is a fixed value called the handover threshold. For successful handover, a channel must be granted to handover request before the power received by the MS reaches the receiver's threshold. The handover area is the area where the ratio of received power levels from the current and the target BS's is between the handover and the receiver threshold. Each handoff requires network resources to reroute the call to the new base station. Minimizing the expected number of handoffs minimizes the switching load. Another concern is delay. If the handoff does not occur quickly, the quality of service may degrade below an acceptable level. Minimizing delay also minimizes co-channel interference. During handoff there is brief service interruption. As the frequency of these interruptions increases the perceived QoS is reduced. The chance of dropping a call due to factors such as the availability of channels increases with the number of handoffs attempts. As the rate of handoff 
increases, handoff algorithms needs to be enhanced so that the perceived QoS does not degrade and the cost to cellular infrastructure does not increase.

\subsection{Performance metrics for Handovers}

The following are the performance metrics for handovers.

Call blocking probability - The probability that a new call attempt is blocked.

$>$ Handoff blocking probability - The probability that a handoff attempt is blocked.

$>$ Handoff probability - The probability that while communicating with a particular cell, an ongoing call requires a handoff before the call terminates. This metric translates into the average number of handoffs per cell.

$>$ Call dropping probability - The probability that a call terminates due to handoff failure. This metric can be derived directly from the handoff blocking probability and the handoff probability.

$>$ Rate of handoff - The number of handoff per unit time.

$>$ Duration of interruption - The length of time during handoff for which the mobile terminal is in communication with neither base station.

$>$ Delay - The distance the mobile user moves from the point at which, the handoff should occur to the point at which it does.

\subsection{Schemes to handle Handoff failure}

In mobile cellular systems, especially when relatively small cell or micro cells are used, the handover procedure has a significant impact on the system's performance. Channel assignment strategies with handover have been proposed in order to decrease the probability of forced termination. Two generic handover schemes are:

1. Reserving a number of channels exclusively for handovers:

The so-called "guard channel" concept offers a means of improving the probability of successful handover by simply reserving a fixed or dynamically adjustable number of channels exclusively for handover requests. Reserving channels for handovers means less channels been granted to originating calls so that the total carried traffic is reduced.

2. Queuing handover request:

Queuing handover requests, with or without reserving channels for handovers, is another method of reducing the probability of forced termination at the expense of an increased call blocking and a decrease in the ratio of carried -to-admitted traffic.

\section{LITERATURE SURVEY}

Existing work addresses the concept of Fixed Channel Allocation Scheme (FCA) where there are no separate channels allocated for handoffs. The available channels are shared by both new originating calls and handoff calls in first come first serve basis [8]. Here handoff request and new call request are dealt with equality. The cell doesn't consider the difference between Handoff request and new call request. It is intuitively clear that the termination of an ongoing call due to 
handoff failure is less desirable than the new call blocking. Hence the Quality of Service is not satisfied because the handoff blocking rate is as same as new call blocking rate [11]. Many papers in the literature of related work address the categorization of the schemes based on guard channel concept [1][10]. The so called "Guard-channel" (GC) concept offers a means of improving the probability of a successful handoff by reserving a certain number of channels allocated exclusively for handoff requests. The remaining channels can be shared equally between handoff requests and new calls. Allocating Guard channels for Handoff improves the overall throughput which was discussed in our previous papers [12][13]. If the guard channel number is too big, the new call blocking rate will be high because several channels are set aside for handoff requests even when the traffic load is low. In this case, the resources are wasted by not serving either for handoff request or new call request. If the number is too small, the handoff blocking rate can't be guaranteed under high traffic load. So this scheme enhances the QoS by reducing the handoff blocking rate in a stable traffic load. While when the traffic load is changing periodically or dynamically due to big event or working rush hours, it is not flexible enough to get good QoS.

\section{Proposed WORK}

In this paper, the authors devise a scheme called Dynamic Guard Channel Allocation with Channel Borrowing Strategy (DGCA-CBS). In this scheme the channels for handoff requests are dynamically allocated based on the observation of certain past period of time in the network. Also, this scheme introduces a concept of channel borrowing in which the guard channels could be allocated to the new originating calls if they are unused. When a new originating call arises and if all the available channels are occupied it will check for the guard channels. If it is unused the new call will occupy the guard channel. The main aim is to utilize the available resources efficiently and also to balance the load in the network traffic. The following section presents the proposed scheme.

\subsection{Dynamic Guard Channel Allocation with Channel Borrowing Strategy (DGCA- CBS)}

A call being forced to terminate during the service is more annoying than a call being blocked at its start. Hence the handoff call blocking probability is much more stringent than new call blocking probability. Therefore, in the proposed scheme, priority is given to handoff requests by assigning $\mathbf{S}_{\mathbf{R}}$ channels exclusively for handoff calls among the $\mathbf{S}$ channels in a cell. The remaining $\mathbf{S}_{\mathbf{C}}\left(=\mathbf{S}-\mathbf{S}_{\mathbf{R}}\right)$ channels are shared by both originating calls and handoff requests. The selection of number of guard channels exclusively for handoff call is essentially important factor to get good Quality of Service. For different type of traffic load and mobility factor, different number of guard channels is needed to be allocated. The number of guard channels can't be fixed when the traffic load is changing with the time. Hence the guard channel allocation is dynamically changed by monitoring the traffic condition for certain time period. Also, the proposed scheme introduces the concept of channel borrowing strategy. In this strategy the exclusive guard channels are borrowed and allocated to new originating calls if the channel is unused. An originating call is blocked if channel is not available in the target cell. The channel allocation model is shown in figure 4 . 


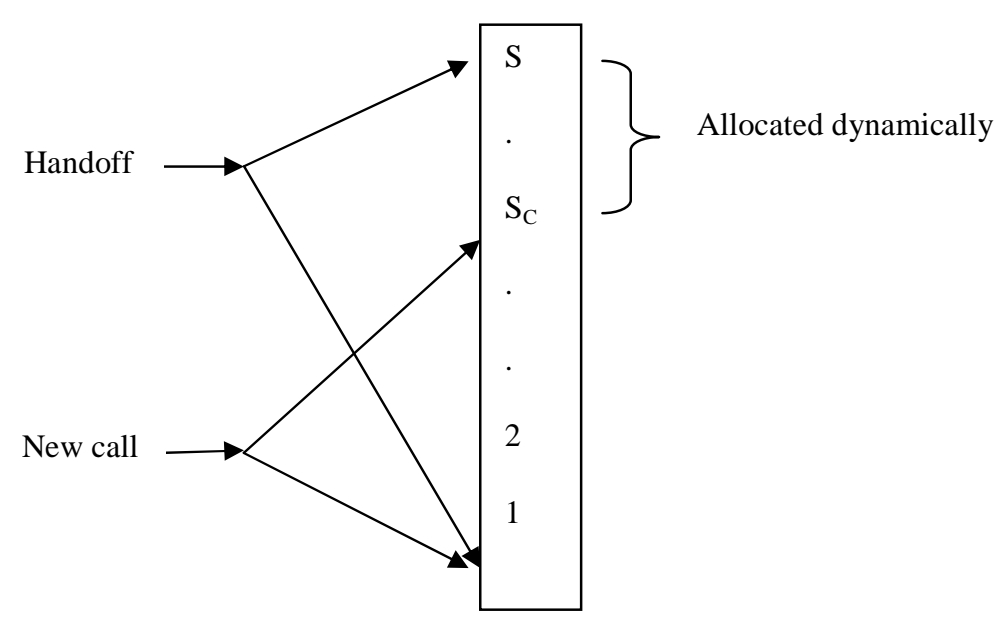

Figure 4. Dynamic Channel allocation model with priority for handoff calls.

In the proposed scheme, when a new call originates the BS will check for available channels in $S_{C}$. If channel is not free in $S_{C}$ it will check for non utilized channels in $S_{R}$. If channels are available in $S_{R}$ then BS borrows the channels and assigns it to the new originating calls. The BS monitors the traffic for certain time period and records it. Based on the network traffic the BS dynamically allocates the number of Guard channels exclusively for handoffs. Generally the number of handoff requests will be high in peak hours and is low during night and non peak hours. Hence based on the observed measurements the BS reallocates the guard channel. The entire operation is controlled by BS and MSC and hence the scheme is Network controlled handoff scheme [2][14].

As the number of Guard Channels allotted plays a vital role to the key performance, it is dynamically altered every specific time period say t. In this approach the number of guard channels which is to be allocated is determined through optimizing certain performance goal with service quality constraints. When a base station experiences high handoff blocking rate, the number of guard channels will be increased until the handoff blocking rate drops to below its threshold. When a base station does not get to use a significant portion of the guard channels over a period of time, the number of guard channels is gradually decreased until most of the guard channels are used frequently. By doing this, the handoff blocking rate is controlled to close to its threshold. The flow chart for the proposed scheme is depicted in figure 5. 
International Journal on AdHoc Networking Systems (IJANS) Vol. 2, No. 3, July 2012

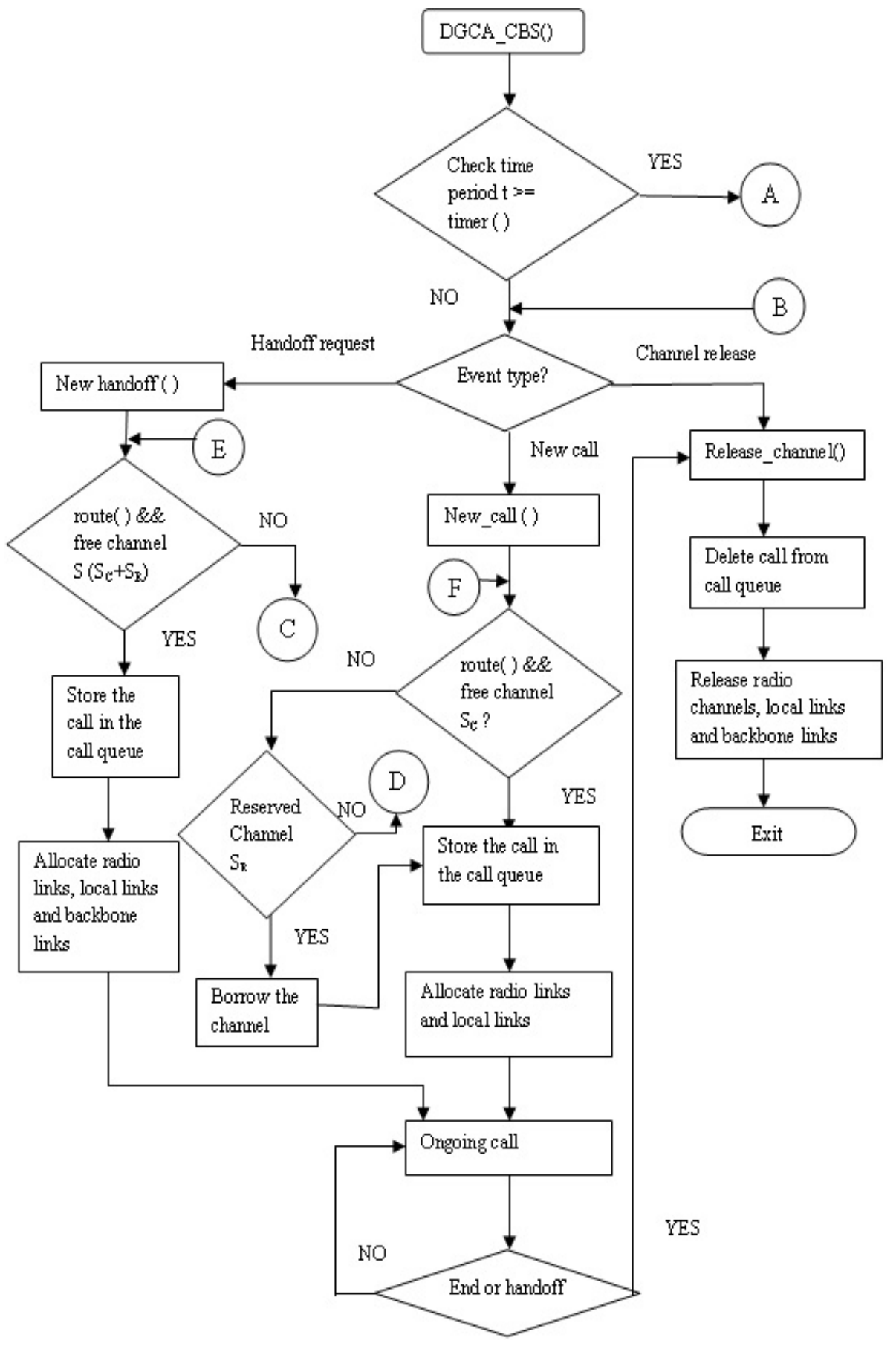


International Journal on AdHoc Networking Systems (IJANS) Vol. 2, No. 3, July 2012

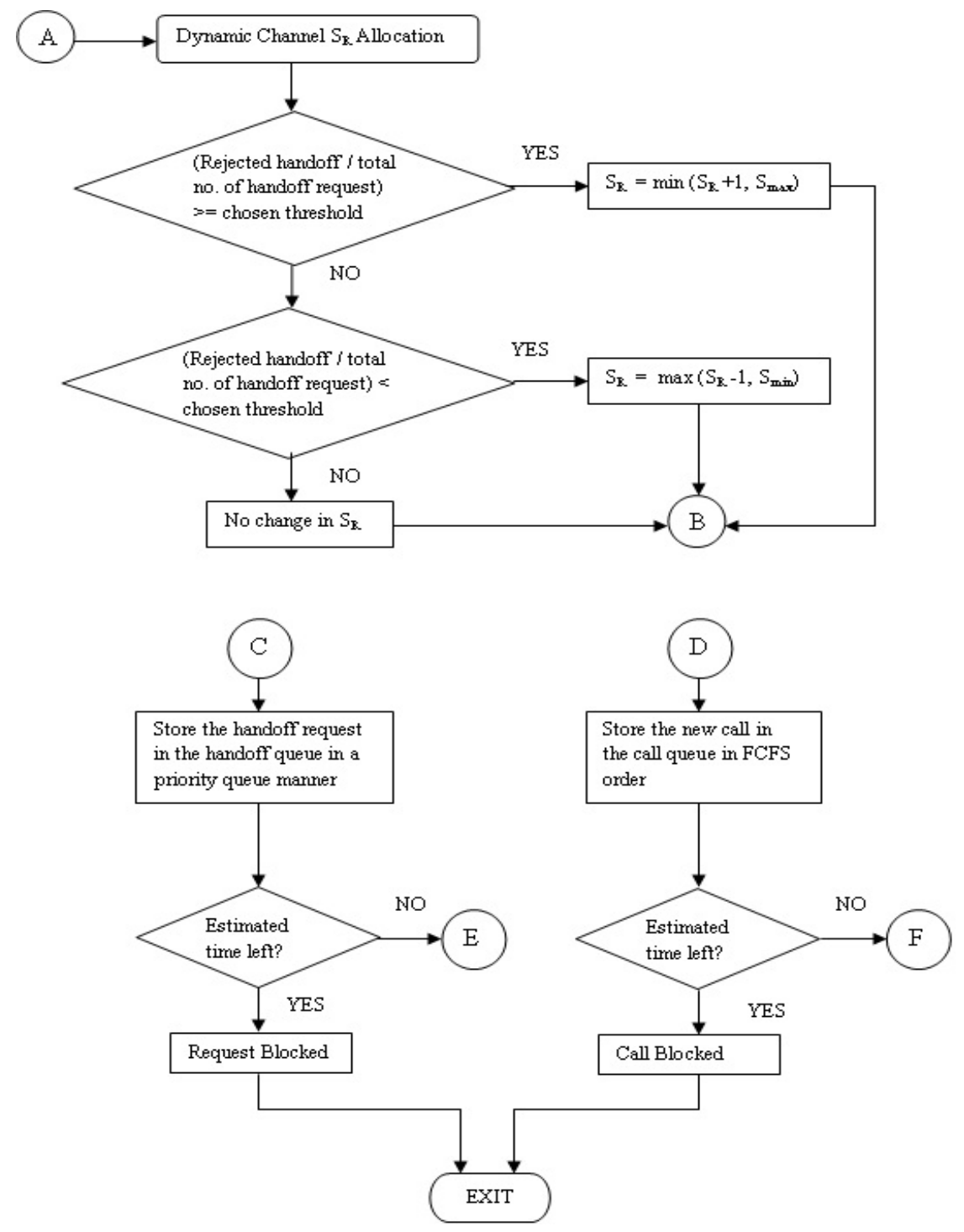

Figure 5. Flowchart represents DGCA-CBS

\section{SimUlation}

The Simulation is carried out with two models of network as Simulation model I and Simulation model II. In Simulation model I, the authors simulate the traffic in six cells as a part of full network. As shown in figure. 6, the authors consider three ATM switches from 0 to 2. BTS0 and BTS1 form a cluster and are connected to ATM switch 0, BTS2 and BTS3 form a cluster and are connected to ATM switch 1 and BTS4 and BTS5 form a cluster and are connected to ATM switch 2. ATM switches are connected by backbone links illustrated in the figure 6 .

In simulation model II, the authors simulate the traffic in twelve cells as a part of full network. As shown in figure 7, the authors consider six ATM switches from 0 to 5. BTS0 and BTS1 form a cluster and are connected to ATM switch 0, BTS2 and BTS3 form a cluster and are connected to ATM switch 1, BTS4 and BTS5 form a cluster and are connected to ATM switch 2, BTS6 and 
BTS7 form a cluster and are connected to ATM switch 3, BTS8 and BTS9 form a cluster and are connected to ATM switch 4, BTS10 and BTS11 form a cluster and are connected to ATM switch 5. The ATM switches are interconnected by seven-backbone link.

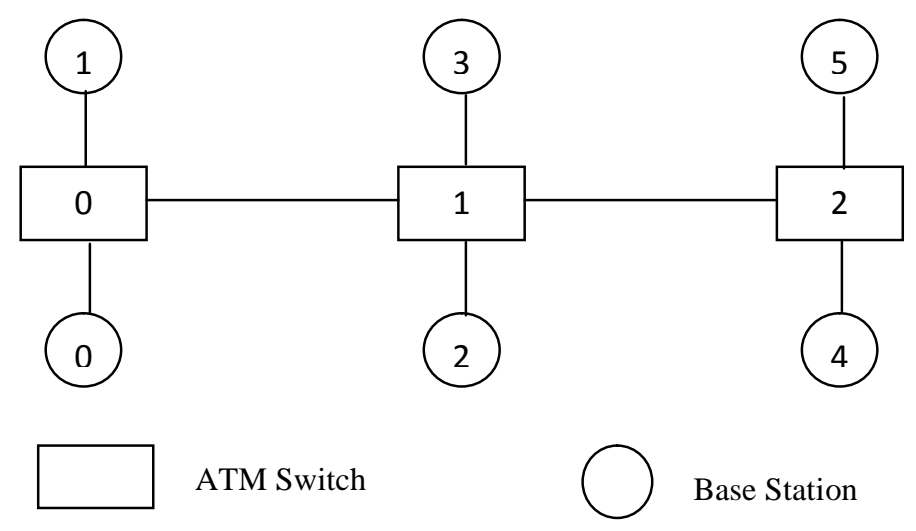

Figure 6. ATM-based cellular PCN model for simulation I.

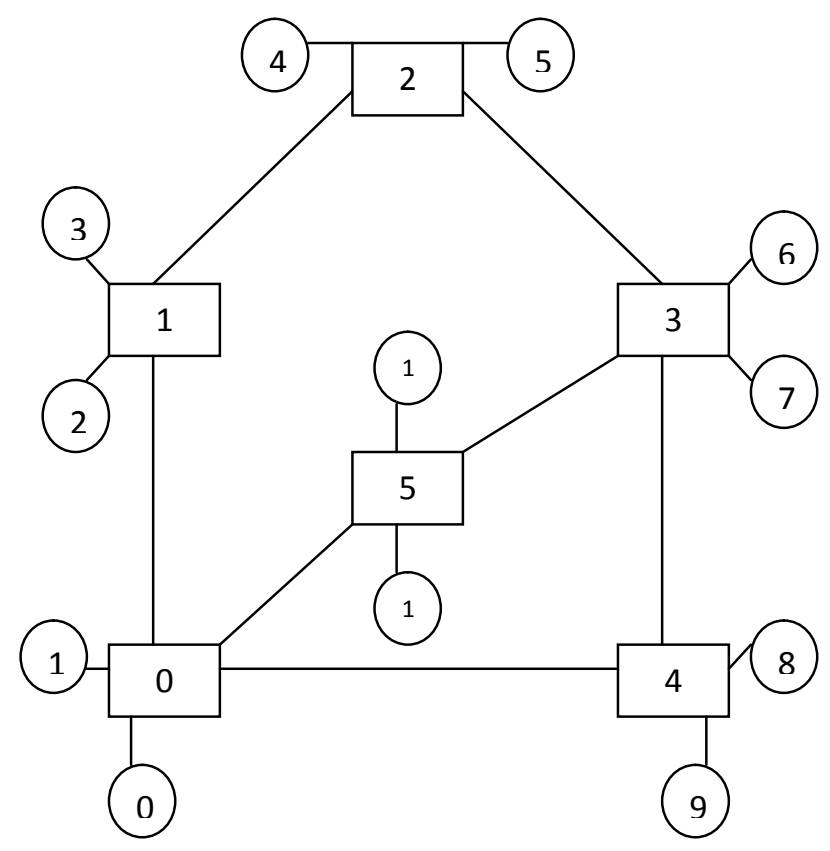

Figure 7. ATM based cellular PCN model for simulation II.

Mobile Terminals (MT) may handover to cells, which are inside or outside the clusters under simulation; this allows a radio channel release in that BTS and may or may not affect the load in the backbone link, depending on the network topology and the destination. In this paper, the authors consider this has no effect on the backbone link. Each BTS has a limited number of radio channels. Local link has a number of wired channels, which is equal to radio channels. Backbone link has a limited number of wired channels. For simplicity, the roaming of the mobile terminal in the test cells only is considered. The destination of the call is important to determine the need for 
local and backbone links; therefore the authors define three call types according to its destination, with probability of occurrence in the simulated environment as follows:

- In Cell Call: In which the call source and destination are in the same cell, the probability of this call is $\mathrm{P}_{\text {cell }}=1 / 7$, here MT consumes only one radio channel.

- In Cluster Call: In which the call source and the destination are in the same cluster, but different cells. Probability of this call is $P_{\text {cluster }}=1 / 7$. Here MT consumes one radio channel and one local link channel.

- Out Cluster Call: In which the call source and the destination are in different clusters. Probability of this call is $\mathrm{P}_{\text {backbone }}=5 / 7$, Here MT consumes one radio channel, one local link and backbone link channels depending on the path allocated.

Note that, there is no competition for local links. If the radio channel is available, then local link is granted, because only the user in the cell may use the local link channel. The call will occupy resources according to the probability above. If there are not enough resources available then the call will be blocked. The new call may handover to the neighboring cell releasing the radio channel and local link channel, but may or may not release the backbone link depending on the destination and rerouting algorithm used.

\title{
5.1 Simulation Parameters
}

Following simulation parameters are used.

busy_channels : Number of channels occupied by calls.

next_event_type : Type of next event New call, New handover, Channel release

total_calls : Number of calls generated in or handed to the cell.

new_success : New calls which have been assigned channels by the BS.

ho_success : Handovers which have been assigned channels by the BS

ho_fail : Handovers which have not been assigned channels.

blocked : New calls which have not been allocated channels.

incell_success : New incell call or handover which have been assigned channels.

incell_blocked : New incell call or handover which have not been assigned channels.

incluster_success : New incluster call or handover which have been assigned channels.

incluster_blocked : New incluster call or handover which have not been assigned channels. outcluster_success: New outcluster call or handover which have been assigned channels outcluster_blocked: New outcluster call or handover which have not been assigned channels.

call_type : Type of call; Incell, Incluster, Outcluster

BTS_index : Index of Base Station whose event will occur.

Capacity : : Load which a backbone link can handle.

next_call : Time at which next new call will be generated.

next_event_time : Time at which next event will occur.

next_handover : Time at which next new handover will be generated.

ho_delay : Time for which a handover is stored in the handover queue.

miat : Mean inter arrival time. Time difference between successive calls

hmiat :Handoff miat. Time difference between successive handover.

\subsection{Data Structure and Functions}

\author{
struct calls \\ \{ \\ float atime;
}




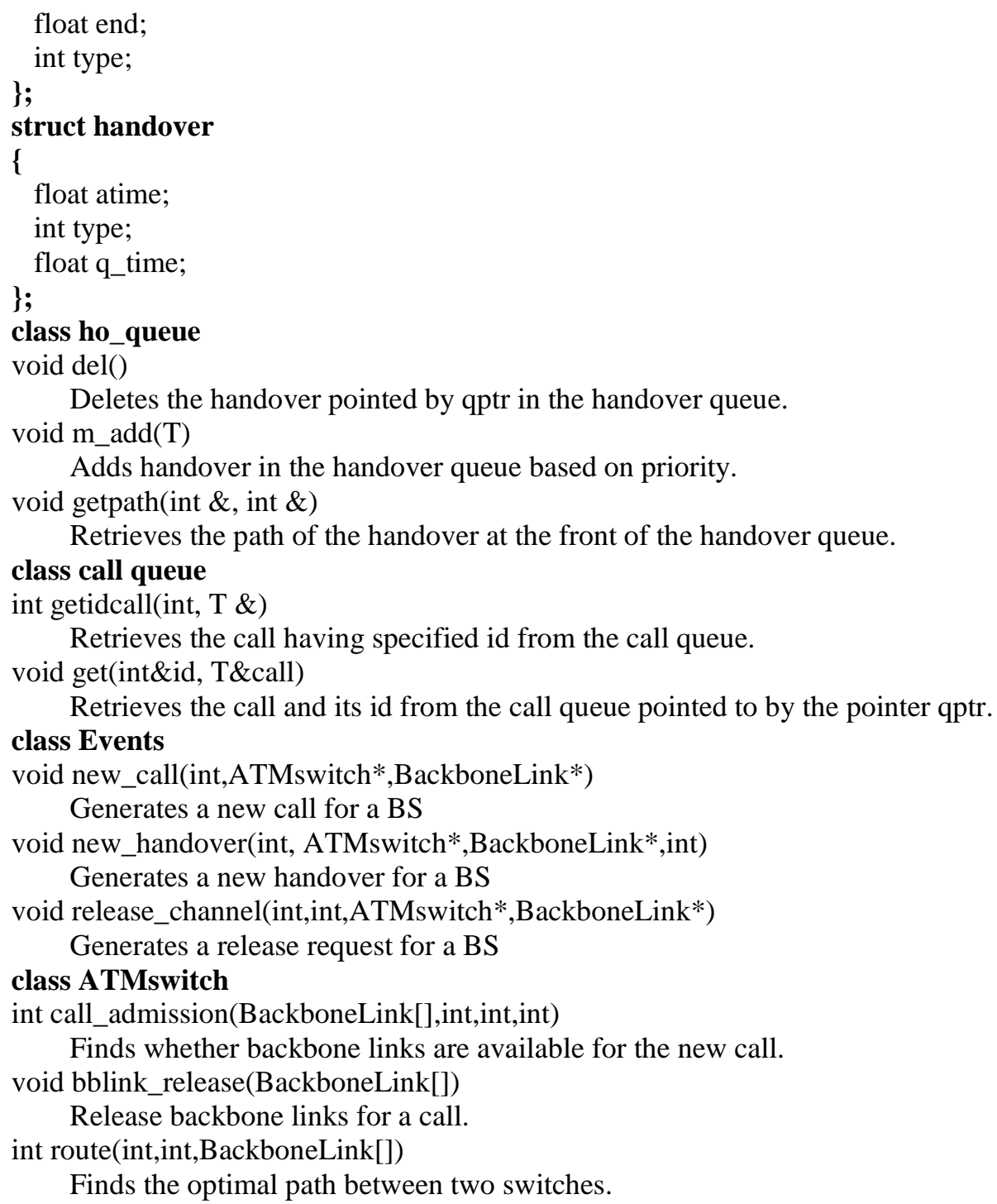

\section{RESULTS AND DISCUSSION}

Simulation is the best method to indicate that a solution is likely to work in an environment where the real-life network is not easily available. This attempts to explain observed behavior using a set of simple and understandable rules. These rules can be used to predict the outcome of experiment involving the given physical situation. In this research work, the DGCA-CBS method is simulated for a part of full network with two different models as explained in the previous section. The simulation program is implemented in Turbo $\mathrm{C}++$, version 3.0, and run under MS DOS 6.2 environment. Object oriented approach is adopted to simulate the real world environment. Results are directed to a text file and graphs for the same are plotted using MATLAB. The simulation program implements the system characteristics with all the necessary QoS parameters to determine the various blocking probabilities. The simulator generates numerical data pertaining to offered load and blocking probabilities. The generated data represents traffic rate in a real life network, an indication of the system state for a time period exhibited by the values of new call rejection probability and handoff call blocking probability. The results for the three switch ATM 
network and the six switch ATM network have been computed. The result comprises of comparison between the four schemes namely Fixed Channel Assignment Scheme, Static Guard Channel Allocation Scheme, Dynamic Guard Channel Allocation without Channel Borrowing Scheme and Dynamic Guard Channel Allocation with Channel Borrowing Strategy. The graph from figure 8 to figure 11 shows the comparative study of these four schemes. Initial starting parameters are listed below.

Number of Radio Channels = $\quad 30$ per cell.

Number of Local Link Channels $=30$ per cell.

Average time for a new call $=60 \mathrm{sec}$.

Average time for a handover call $=30 \mathrm{sec}$.

Maximum handover queue time $=10 \mathrm{sec}$.

Capacity of Backbone Links $=50$ calls.

Number of Static Guard Channels $=5$

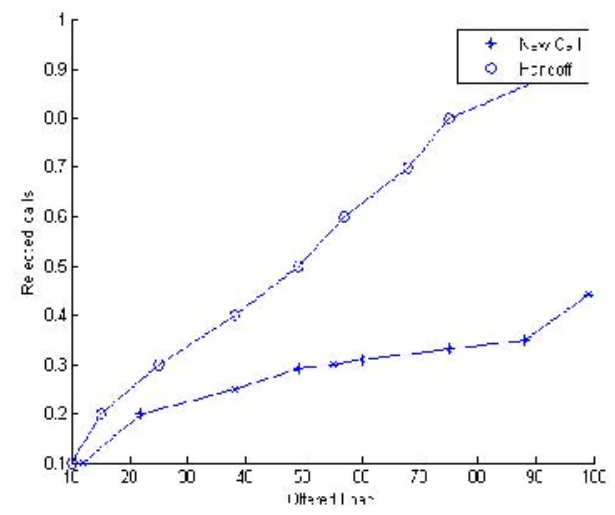

Figure 8. Fixed Channel Assignment scheme

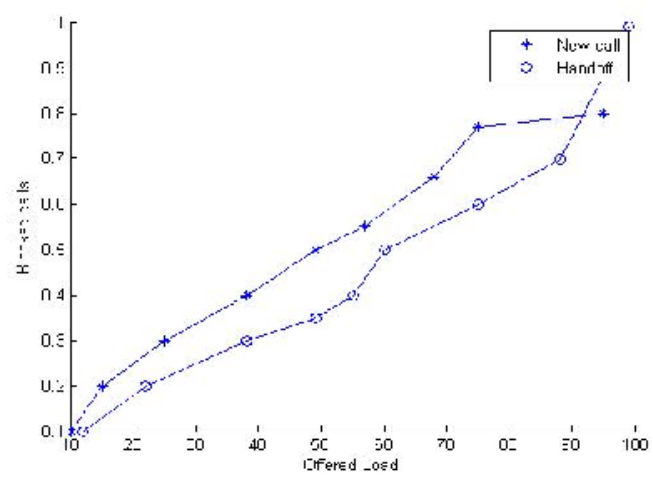

Figure 9. Static Guard Channel Allocation scheme 
International Journal on AdHoc Networking Systems (IJANS) Vol. 2, No. 3, July 2012

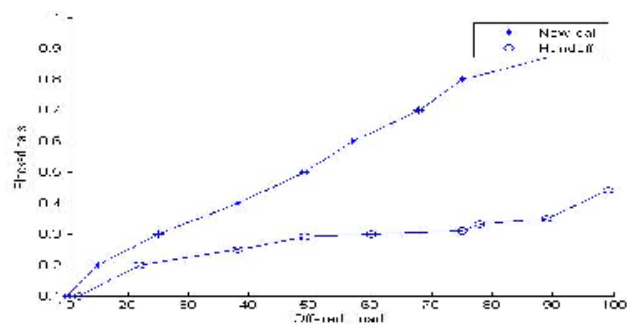

Figure 10. Dynamic Guard Channel Allocation without Channel Borrowing scheme

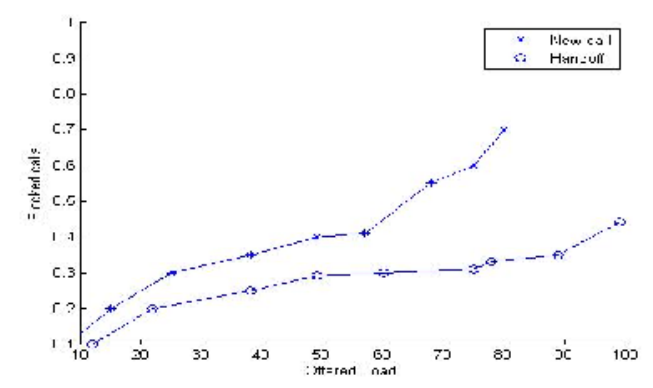

Figure 11. Dynamic Guard Channel Allocation with Channel Borrowing strategy (DGCA-CBS)

Figure 8 shows the simulated output of the Fixed Channel Assignment scheme where there is no separate guard channels allocated for handoff request. From the graph it is clear that handoff request rejection rate is high than the new call rejection rate. Hence the performance is not satisfactory as handoff requests should be given higher priority. Figure 9 shows the graph of Static Guard Channel Assignment scheme. This graph proves that the handoff blocking rate is reduced but the new call blocking rate is increased. Figure 10 shows the output of the Dynamic Guard channel allocation without channel borrowing scheme. From the graph it is apparant that the handoff blocking rate is considerably less than the new originating call blocking rate and the new call blocking rate is also better than graph in figure 9 . Figure 11 shows the simulated output of the proposed scheme, Dynamic Guard Channel Allocation scheme with Channel Borrowing Strategy. From the graph it is evident that there is no difference for handoff calls when compared to the graph in figure 10, but for new call there is an improvement. The simulated output shows that there is a significant improvement in reducing the handoff request blocking rate and new call blocking rate in the proposed scheme DGCA-CBS, compared to the existing schemes. Hence there is a tradeoff.

\section{CONCLuSiON}

In wireless mobile networks, as the cell size becomes smaller, handoffs occur more frequently. Careful design of call admission control scheme guarantees QoS to the mobile users. In this paper, the authors approached the channel allocation control scheme in a new way. A new Dynamic Guard Channel Allocation with Channel Borrowing Strategy (DGCA-CBS) is designed and implemented. The objective of this scheme is to utilize the available channel effectively. The main disadvantage of Dynamic Guard channel allocation scheme without channel borrowing strategy is new calls starving for channels while the guard channels remain unused. The concept of channel borrowing is incorporated in the proposed scheme which promises improvement in the new call blocking rate. 


\section{REFERENCES}

[1] Guerin R, "Queuing Blocking System with Two Arrival Streams and Guard Channels", IEEE Transactions on Communications, 1998.

[2] Yi Zhang and Derong Liu, "An Adaptive Algorithm for Call Admission Control in Wireless Networks", IEEE 2001

[3] QING-AN-ZENG and DHARMA P.AGARWAL, "Handbook of Wireless Networks and Mobile Computing",John wiley \& sons, Inc., 2002

[4] A. Iera, A. Molinaro and S. Marano, "Handoff Management with Mobility Estimation in Hierarchical Systems", IEEE Transactions on Vehicular Technology, vol. 51, Sept. 2002, pp. 915-934.

[5] A. Hills and J. Schlegel, "Rollabout: A wireless design tool,” IEEE Commun. Mag., vol. 42, pp. 132138, February 2004.

[6] F. Siddiqui and S. Zeadally, "Mobility Management across Hybrid Wireless Networks: Trends and Challenges," Computer Communications, vol. 29, no. 9, pp. 1363-1385, May 2006.

[7] Nasser.N, Hasswa. A and Hassanein. H, "Handoffs in Fourth Generation Heterogeneous Networks", IEEE Communications Magazine, vol. 44, pp. 96-103, 2006

[8] Rami Tawil, Jaques Demergian, Guy Pujolle, "A Trusted Handoff Decision Scheme for the Next Generation Wireless Networks", IJCSNS International Journal of Computer Science and Network Security, VOL.8 No.6, June 2008

[9] Yoo, S.-J.,et al., "Analysis of fast handover mechanisms for hierarchical mobile IPv6 network mobility", Wireless Personal Communications, 48(2), 215-238., 2009.

[10] Ojesanmi O. A, Ojesanmi A. and Makinde O, "Development of Prioritized Handoff Scheme for Congestion Control in Multimedia Wireless Network", Proceedings of the World Congress on Engineering, Vol I, 2009

[11] C. B. Akki1, S. M. Chadchan2, "The Survey of Handoff Issues in Wireless ATM Networks", International Journal of Nonlinear Science, Vol.7, 2009

[12] Alagu.S and Meyyappan.T, "Analysis of Algorithms for Handling Handoffs in Wireless Mobile Networks", International Journal of P2P Network Trends and Technology- Volume1Issue2- 2011 ISSN: 2249-2615, pg 49-56

[13] Alagu S and Meyyappan T, "Analysis of Handoff Schemes in Wireless Mobile Network", IJCES International Journal of Computer Engineering Science", Volume1 Issue2, November 2011 ISSN : 2250:3439, pg $1-12$

[14] V. S. Kolate, G. I. Patil, A. S. Bhide, "Call Admission Control Schemes and Handoff Prioritization in 3G Wireless Mobile Networks", International Journal of Engineering and Innovative Technology (IJEIT) Volume 1, Issue 3, March 2012

\section{Authors}

Mrs.S.Alagu, M.Sc., M.Phil., currently, Ph.D. Research Scholar in Department of Computer Science and Engineering, Alagappa University, Karaikudi. She has a teaching experience of 10 years. She has published research papers in National and International Journals and Conferences. Her research area includes Wireless Mobile Networks.

Dr. T. Meyyappan M.Sc., M.Phil., M.B.A., Ph.D., currently, Professor, Department of Computer Science and Engineering, Alagappa University, Karaikudi, TamilNadu. He has obtained his Ph.D. in Computer Science in January 2011 and published a number of research papers in National and International journals and conferences. He has been honored with Best Citizens of India Award 2012 by International Publishing House, New Delhi. He has developed Software packages for Examination, Admission Processing and official Website of Alagappa University. His research areas include

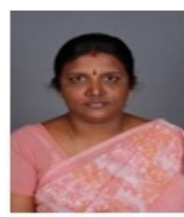
Operational Research, Digital Image Processing, Fault Tolerant computing, Network security and Data Mining. 